Case Report

\title{
Diffuse Cutaneous Mucinosis in Dermatomyositis: A Case Report and Review of the Literature
}

\author{
Alexandra Caitlin Perel-Winkler ${ }^{1}$ and Chris T. Derk ${ }^{2}$ \\ ${ }^{1}$ St. Luke's-Roosevelt Hospital Center, New York, NY 10025, USA \\ ${ }^{2}$ Division of Rheumatology, University of Pennsylvania, One Convention Boulevard, 8th Floor Penn Tower, \\ Philadelphia, PA 19104, USA \\ Correspondence should be addressed to Chris T. Derk; chris.derk@uphs.upenn.edu
}

Received 29 July 2014; Accepted 1 November 2014; Published 18 November 2014

Academic Editor: Ravi Krishnan

Copyright (C) 2014 A. C. Perel-Winkler and C. T. Derk. This is an open access article distributed under the Creative Commons Attribution License, which permits unrestricted use, distribution, and reproduction in any medium, provided the original work is properly cited.

\begin{abstract}
We present the case of a patient with dermatomyositis and diffuse cutaneous mucinosis and give an up-to-date detailed review of all the published cases in the English literature describing the demographics, clinical picture, pathology management, and outcomes of this unique group of patients.
\end{abstract}

\section{Introduction}

Mucin (hyaluronic acid complex) is a protein normally found as part of the dermal connective tissues and it is produced by mast cells and fibroblasts. As hyaluronic acid holds water, in disease states where mucin production is increased, the dermal connective tissue becomes swollen and is described as myxedematous. It is not uncommon to have findings of microscopic cutaneous mucinosis in the setting of collagen vascular diseases and mucin deposition in the correct clinical setting can be considered as histologic evidence of dermatomyositis (DM) [1]. Clinically evident forms of mucinosis have been described in hypothyroidism, thyrotoxicosis, scleromyxedema associated with monoclonal gammopathies, scleredema related to diabetes, and lichen myxedematosus. Cases of secondary cutaneous mucinosis have been described in systemic lupus erythematosus, systemic sclerosis, and dermatomyositis, albeit infrequently [2-8]. We present a case of dermatomyositis with evidence of diffuse cutaneous mucinosis in a patient recently treated for nonsmall cell lung cancer (NSCLC) without evidence of recurrence.

\section{Case}

A 57-year-old man with chronic obstructive lung disease, hypothyroidism, gastroesophageal reflux disease, and a prior history of NSCLC developed a pruritic, confluent, violaceous rash after cancer treatment. The patient was diagnosed with NSCLC in 2011 and was treated with paclitaxel and carboplatin and adjunctive radiation, with a restaging PET/CT scan showing excellent response. Four months after the completion of chemotherapy and radiation therapy the patient presented complaining of a pruritic rash. The rash first appeared on his hands and was noted to be consistent with Gottron's papules. Over the next nine months the rash worsened, and the patient developed violaceous erythema on his upper chest and back. Erythematous patches with white macules then developed on his lower legs, thighs, and buttocks. Three years after the treatment of his cancer, the patient had a diffuse, scaly, and erythematous rash on his arms (Figure 1), legs, buttocks, abdomen, neck, and face (Figure 2) with evidence of white macules (Figure 3) most prominent on the upper and lower extremities. Initial concern was for recurrence of his cancer; however, full body PET-CT revealed no new or active cancer. Skin biopsies showed evidence of interface dermatitis with sections of hyperkeratosis, mild spongiosis, interface vacuolar change, and dermal mucinosis without involvement of the panniculus or fascia (Figures 4 and 5). Muscle enzyme tests showed a normal creatinine phosphokinase level but an elevated aldolase at 9.5 U/L. A later full thickness biopsy performed showed evidence of interface dermatitis with mucin deposition. Two muscle biopsies were 


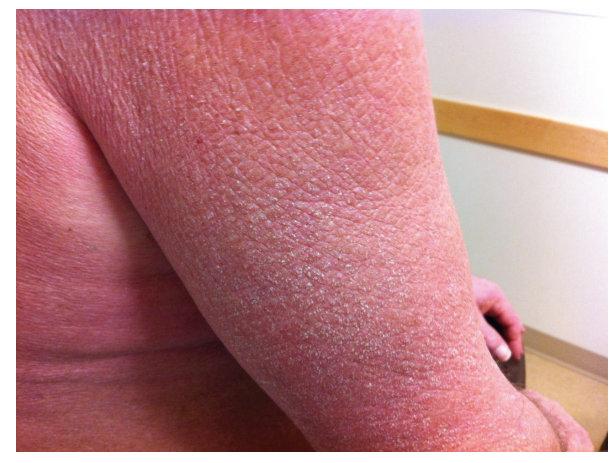

FIGURE 1: Cutaneous mucinosis: violaceous, scaly, and erythematous rash of the right arm.

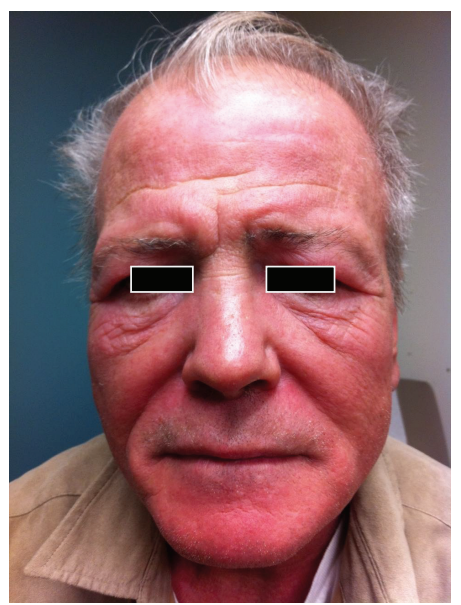

Figure 2: Cutaneous mucinosis: diffuse erythematous, violaceous rash of the face.

performed and HLA1 staining showed diffuse labeling of the sampled myofibers. Only one necrotic myofiber was isolated; otherwise the specimens were largely normal without diffuse myofiber necrosis, inflammation, or definite vacuolation. An MRI of the patient's femurs showed hyperenhancement in the obturator internus and externus muscles bilaterally and the proximal hamstrings (right greater than left), indicating some degree of inflammation. Immunoserologic results included a positive ANA of 1:640 with a speckled pattern and a positive Smith antibody (Ab). Of the myositis autoantibody panel, anti-Ku and anti-U1RNP were found to be positive. Other labs included a normal TSH and a slightly elevated gammaglobulin fraction of $1.7 \mathrm{~g} / \mathrm{dL}$ (reference range $0.7-1.2 \mathrm{~g} / \mathrm{dL}$ ) with a normal immunofixation.

Dermatomyositis with cutaneous mucinosis was diagnosed in light of the physical exam findings, MRI evidence of inflammation, evidence of interface dermatitis, and mucin deposition on the skin biopsies and positive serologies. The demonstration of mucinosis without fibroblastic proliferation or dermal thickening supported a diagnosis of cutaneous mucinosis as opposed to scleromyxedema or systemic sclerosis.

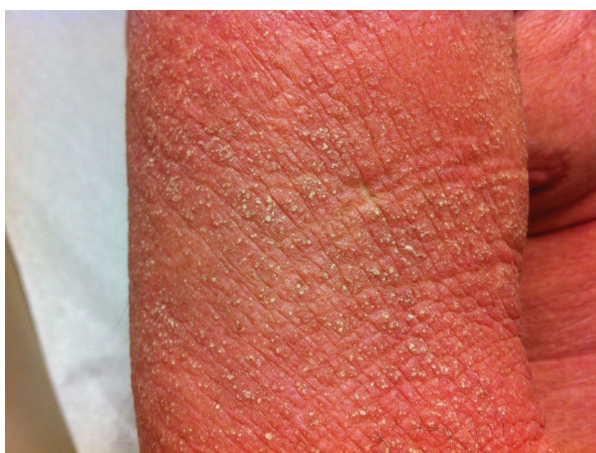

FIgURE 3: Cutaneous mucinosis: diffuse, scaly, and erythematous rash with white macules.

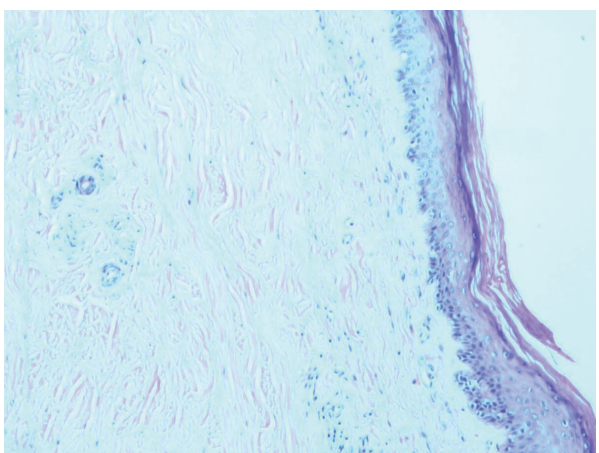

FIGURE 4: Skin biopsy: colloidal iron with hyaluronidase $\times 100$. Dermal mucin deposition without fibroblast proliferation, with interface vacuolar changes.

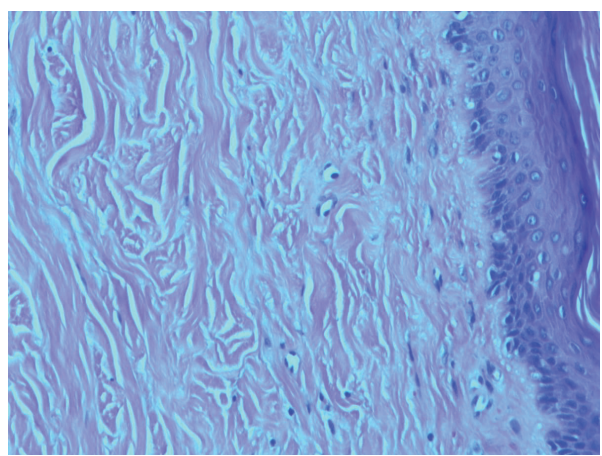

FIGURE 5: Skin biopsy: colloidal iron $\times 200$ : dermal mucin depositions without fibroblast proliferation.

Prior to presentation at our clinic, 3 years after the initial symptoms began, the patient had tried multiple medical treatments. He was initially treated with $5 \mathrm{mg}$ of oral prednisone, which was quickly increased to $20 \mathrm{mg}$ without success. Methotrexate was initiated at $7.5 \mathrm{mg}$ weekly and then titrated to $15 \mathrm{mg}$ weekly without response. Plaquenil $200 \mathrm{mg}$ was tried for 2 months but the patient discontinued the treatment as he felt it had no effect. Once we diagnosed the patient with dermatomyositis and diffuse cutaneous mucinosis, we initiated $60 \mathrm{mg}$ of prednisone per day which was tapered to $40 \mathrm{mg}$ daily two weeks later due to side effects. 
Intravenous Immunoglobulin was initiated at 20 grams for 3 consecutive days every 6 weeks. At 3-month follow-up, the patient reported significant improvement in the amount of erythema and induration especially in the upper extremities and a decrease in the white macular lesions.

\section{Discussion}

3.1. Dermatomyositis and Cutaneous Mucinosis. Dermatomyositis is an inflammatory myopathy, which affects striated muscle and has cutaneous features. Typically a heliotropic rash, Gottron's papules, shawl sign, and erythematous plaques are some of the dermatologic manifestations; however, atypical cutaneous features, including plaque like mucinosis, have also been described [1]. The pathophysiology of dermatomyositis includes the expression of autoantibodies which target protein synthesis or translational particles in the muscle cell which triggers a humoral immune response. Activation of proinflammatory cytokines and chemokines leads to the migration of lymphoid cells to the perimysial and endomysial spaces; complement activation leads to the formation and deposition of membranolytic attack complexes onto endomysial capillaries. The result is microangiopathy and necrosis of endothelial cells leading to perivascular inflammation, muscle ischemia, and muscle fiber destruction $[8,13]$.

Mucin is a mucopolysaccharide produced by fibroblasts and consists of hyaluronic acid and sulfated glycosaminoglycans. Cutaneous mucinosis is subdivided into primary and secondary types; in primary, mucin deposition is the primary histologic feature and secondary, where mucin deposition is an additional finding to a primary clinicopathologic setting. Cutaneous manifestations of mucin can be focal or diffuse and are described as dermal or epidermal (follicular) [9, 14]. The pathophysiology of increased mucin deposition in connective tissue diseases is not completely understood and it is a rare finding. It is postulated that substances circulating in the serum, such as immunoglobulins, autoantibodies, or cytokines, stimulate glycosaminoglycan synthesis by fibroblasts leading to the production of mucin and its deposition in the skin $[8,12]$. Pandya et al. linked the increased level of serum autoantibody titres with an increase in mucin lesions in patients with SLE $[11,15]$. Interleukin-1 and interleukin-6 have also been shown to be elevated in patients with increased dermal mucin production in SLE and DM; however this is nonspecific as interleukins may be raised without evidence of mucinosis [2].

The concept of a hypoxic state contributing to the increased production of mucin has yet to be considered as part of the pathogenesis in DM. In cases of cutaneous mucinosis reported in the setting of venous insufficiency it has been hypothesized that reduced oxygen tension triggers chondrocytes to increase production of hyaluronic acid [16-18]. With perivascular inflammatory infiltrate, capillary obliteration, and myofiber necrosis as known sequelae of DM pathogenesis, it is conceivable that the biologic milieu of DM is hypoxic, and this may be a contributing factor towards mucin production.

Including our patient, there is a total of 12 cases in the English literature describing macroscopically evident cutaneous mucinosis in the setting of dermatomyositis (Table 1). Of these, three cases were associated with malignancy, and one patient had a history of autoimmune thyroiditis, inactive at the time of presentation.

Overall, clinical cutaneous manifestations of mucinous rashes are diverse: Chen, Requena, and Kaufmann describe plaque like skin changes, whereas Wang describes the rash as violaceous; Del Pozo and Johnson describe a distinctly papular rash. Most papers reported classic cutaneous findings of DM alongside the mucinous findings, with Gottron's papules and a heliotrope rash being common. Our patient had the most diffuse mucinous rash of the cases reported, involving the face, chest, back, and all extremities.

In the majority of cases, cutaneous symptoms preceded or occurred simultaneous to muscle weakness. Del Pozo et al. describe mucinous skin changes occurring four years after presentation and treatment of DM, and this is one of two cases where the mucinous skin changes did not resolve $[2,3]$. In general, cutaneous lesions of mucinosis in the setting of DM seem to respond well to treatment when they appear in the early stages of disease. The majority of patients improved with oral steroids \pm azathioprine, with resistant cases improving with IVIG [1]. Only one case did not describe improvement in cutaneous mucinosis despite lack of evidence of malignancy; in this case the mucinosis developed after DM had been successfully treated and did not respond to first line treatment [2]. One case was fatal due to respiratory complications of $\mathrm{DM}$ and recurrent infection due to long-term high dose steroid use [10]. Of note, in the latter two cases IVIG was not utilized per case documentation.

3.2. Dermatomyositis and Malignancy. DM has a clear temporal link with malignancy. Cancer may present in 15-30\% of the adults with DM prior to or at diagnosis or during follow up. DM is most commonly associated with ovarian, breast, lung and colon cancer, melanoma, and non-Hodgkins lymphoma, with adenocarcinomas accounting for $70 \%$ of all associated tumors [19]. The pathophysiology relating DM and malignancy is unproven, but the leading proposed hypothesis is that of an autoimmune paraneoplastic mechanism. Myositis specific antigens (MSA), such as antisynthetase and antisignal recognition particle, have been shown to be expressed at low levels by normal muscles cells and are over expressed during regeneration of muscle fibers during DM [20]. A tumor may overexpress oncoproteins or antigens similar to the myositis antigens, which subsequently stimulate the immune system leading to a lymphocytic reaction causing autoantibody deposition and damage to myofibers [21-23]. Casiola-Rosen showed that solid tumors such as breast and lung may express exact MSA antigens. The damage to muscles causes a release of antigens from the muscle fibers themselves further sensitizing the immune system to the striated muscle. This theory is complemented by the previous theories discussed, correlating serologic antibody titers with DM activity.

As noted previously, malignancy was associated with DM and cutaneous mucinosis in 3 of the 12 cases in the English literature. This proportion of cases with cutaneous mucinosis related to malignancy is proportionate to the number of $\mathrm{DM}$ cases relating to malignancy reported in the literature 


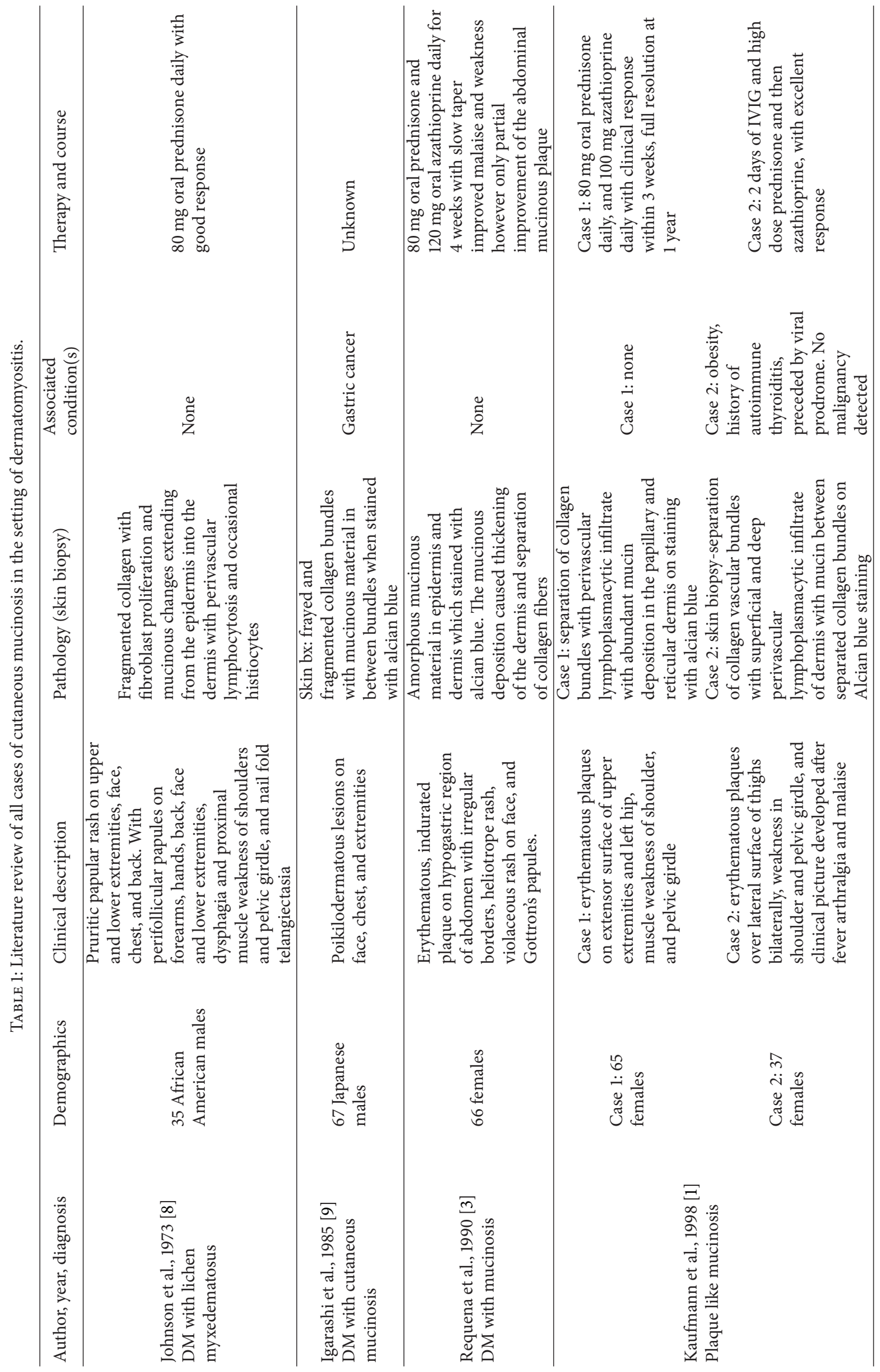




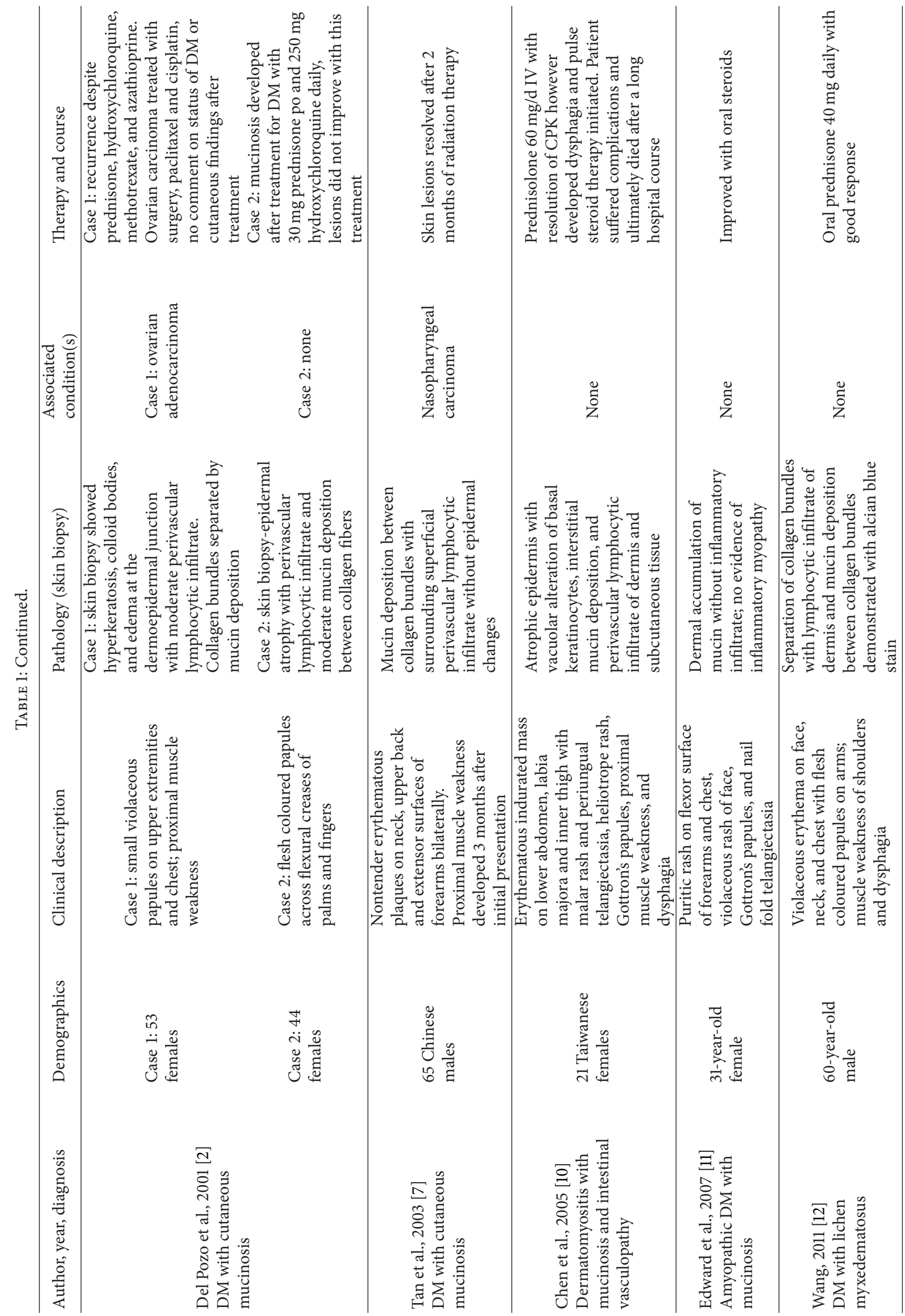


generally (30\%). It is unlikely that the cutaneous mucinosis is independently related to malignancy or that the presence of malignancy increases the chance of cutaneous mucinosis expressed in DM.

\section{Conclusion}

To date there are 12 cases describing macroscopically evident cutaneous mucin in DM. Our case describes a middle aged man with NSCLC in remission presenting with a puritic, diffuse, and violaceous rash. Histologic evidence showed mucin deposition in the dermis without dermal thickening alongside clinical, immunoserologic, and MRI findings consistent with DM, and even though our patient also had serologies suggestive of systemic lupus erythematosus, the predominant clinical picture was that of DM. The patient's cutaneous findings were highly resistant to first and second line treatments and only improved with the initiation of IVIG. While mucin deposition is a common microscopic finding in connective tissue diseases, it is rarely seen macroscopically. The pathophysiologic mechanism of cutaneous mucin production in these clinical scenarios is unclear. The link between hypoxic states and mucin deposition is a new concept, which has not been explored in the setting of dermatomyositis. Of the cases of cutaneous mucinosis and DM in the literature, the majority of cases improved with first line treatment for DM and in resistant cases positive results were seen using IVIG.

\section{Conflict of Interests}

The authors declare that there is no conflict of interests regarding to the publication of this paper.

\section{References}

[1] R. Kaufmann, D. Greiner, P. Schmidt, and M. Wolter, "Dermatomyositis presenting as plaque-like mucinosis," British Journal of Dermatology, vol. 138, no. 5, pp. 889-892, 1998.

[2] J. Del Pozo, M. Almagro, W. Martínez et al., "Dermatomyositis and mucinosis," International Journal of Dermatology, vol. 40, no. 2, pp. 120-124, 2001.

[3] L. Requena, A. Aquilar, and Y. E. Sanchez, "A corrugated plaque on the abdominal wall. Cutaneous mucinosis secondary to dermatomyositis," Archives of Dermatology, vol. 126, no. 12, pp. 1639-1640, 1990.

[4] A. M. Shekari, M. Ghiasi, E. Ghasemi, and Z. A. Kani, "Papulonodular mucinosis indicating systemic lupus erythematosus," Clinical and Experimental Dermatology, vol. 34, no. 8, pp. e558e560, 2009.

[5] M. Allam and M. Ghozzi, "Scleromyxedema: a case report and review of the literature," Case Reports in Dermatology, vol. 5, no. 2, pp. 168-175, 2013.

[6] M. C. Dalakas, "Pathophysiology of inflammatory and autoimmune myopathies," Presse Medicale, vol. 40, no. 4, pp. e237-e247, 2011.

[7] E. Tan, S. H. Tan, and S. K. Ng, "Cutaneous mucinosis in dermatomyositis associated with a malignant tumor," Journal of the American Academy of Dermatology, vol. 48, no. 5, pp. S41S42, 2003.
[8] B. L. Johnson, I. R. Horowitz, C. R. Charles, and D. L. Cooper, "Dermatomyositis and lichen myxedematosus: a clinical, histopathological and electron microscopic study," Dermatologica, vol. 147, no. 2, pp. 109-122, 1973.

[9] M. Igarashi, H. Aizawa, Y. Tokudome, and H. Tagami, "Dermatomyositis with prominent mucinous skin change. Histochemical and biochemical aspects of glycosaminoglycans," Dermatologica, vol. 170, no. 1, pp. 6-11, 1985.

[10] G.-Y. Chen, M.-F. Liu, J. Y.-Y. Lee, and W. Chen, "Combination of massive mucinosis, dermatomyositis, pyoderma gangrenosum-like ulcer, bullae and fatal intestinal vasculopathy in a young female," European Journal of Dermatology, vol. 15, no. 5, pp. 396-400, 2005.

[11] M. Edward, L. Fitzgerald, C. Thind, J. Leman, and A. D. Burden, "Cutaneous mucinosis associated with dermatomyositis and nephrogenic fibrosing dermopathy: fibroblast hyaluronan synthesis and the effect of patient serum," British Journal of Dermatology, vol. 156, no. 3, pp. 473-479, 2007.

[12] S. Wang, "Annular lichen myxedematosus in a patient with dermatomyositis," International Journal of Dermatology, vol. 50, no. 3, pp. 370-372, 2011.

[13] J. T. Kissel, J. R. Mendell, and K. W. Rammohan, “Microvascular deposition of complement membrane attack complex in dermatomyositis," The New England Journal of Medicine, vol. 314, no. 6, pp. 329-334, 1986.

[14] F. Rongioletti and A. Rebora, "Cutaneous mucinoses: microscopic criteria for diagnosis," The American Journal of Dermatopathology, vol. 23, no. 3, pp. 257-267, 2001.

[15] A. G. Pandya, R. D. Sontheimer, C. J. Cockerell, A. Takashima, and M. Piepkorn, "Papulonodular mucinosis associated with systemic lupus erythematosus: possible mechanisms of increased glycosaminoglycan accumulation," Journal of the American Academy of Dermatology, vol. 32, no. 2, pp. 199-205, 1995.

[16] R. Pugashetti, D. C. Zedek, E. V. Seiverling, P. Rajendran, and T. Berger, "Dermal mucinosis as a sign of venous insufficiency," Journal of Cutaneous Pathology, vol. 37, no. 2, pp. 292-296, 2010.

[17] K. Hashimoto, K. Fukuda, K. Yamazaki et al., "Hypoxia-induced hyaluronan synthesis by articular chondrocytes: the role of nitric oxide," Inflammation Research, vol. 55, no. 2, pp. 72-77, 2006.

[18] M. C. Dalakas, "Inflammatory myopathies: management of steroid resistance," Current Opinion in Neurology, vol. 24, no. 5, pp. 457-462, 2011.

[19] S. M. Levine, "Cancer and myositis: new insights into an old association," Current Opinion in Rheumatology, vol. 18, no. 6, pp. 620-624, 2006.

[20] L. Casciola-Rosen, K. Nagaraju, P. Plotz et al., "Enhanced autoantigen expression in regenerating muscle cells in idiopathic inflammatory myopathy," Journal of Experimental Medicine, vol. 201, no. 4, pp. 591-601, 2005.

[21] S. Zampieri, M. Valente, N. Adami et al., "Polymyositis, dermatomyositis and malignancy: a further intriguing link," Autoimmunity Reviews, vol. 9, no. 6, pp. 449-453, 2010.

[22] A. Chakroun, J. Guigay, A. Lusinchi, P. Marandas, F. Janot, and D. M. Hartl, "Paraneoplastic dermatomyositis and nasopharyngeal carcinoma: diagnosis, treatment and prognosis," Annales Francaises d'Oto-Rhino-Laryngologie et de Pathologie CervicoFaciale, vol. 128, no. 3, pp. 148-152, 2011.

[23] P. Ungprasert, N. K. Bethina, and C. H. Jones, "Malignancy and idiopathic inflammatory myopathies," North American Journal of Medical Sciences, vol. 5, no. 10, pp. 569-572, 2013. 


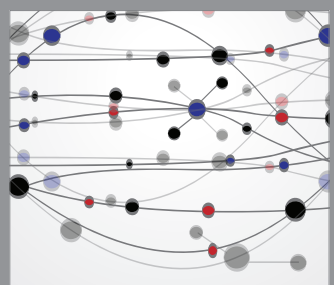

The Scientific World Journal
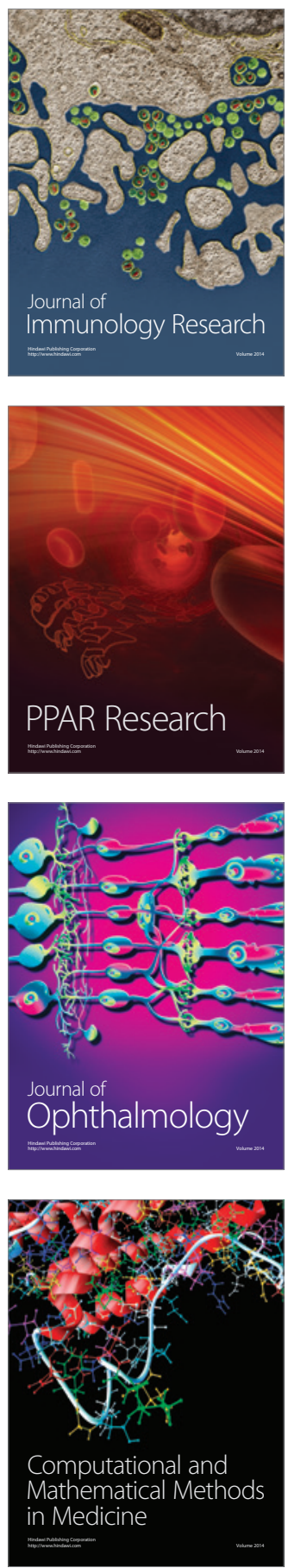

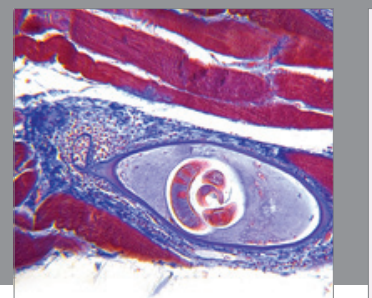

Gastroenterology

Research and Practice
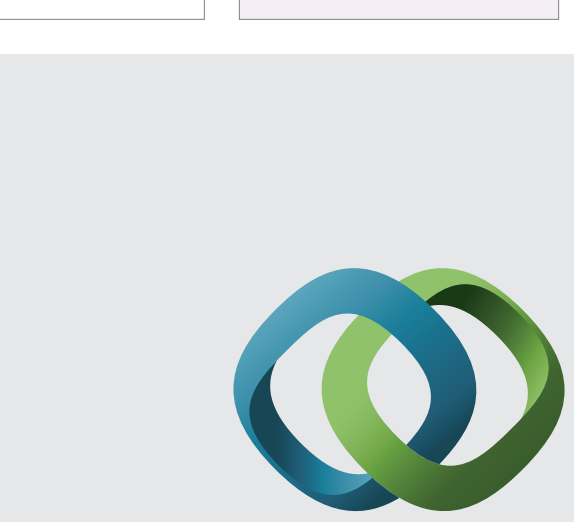

\section{Hindawi}

Submit your manuscripts at

http://www.hindawi.com
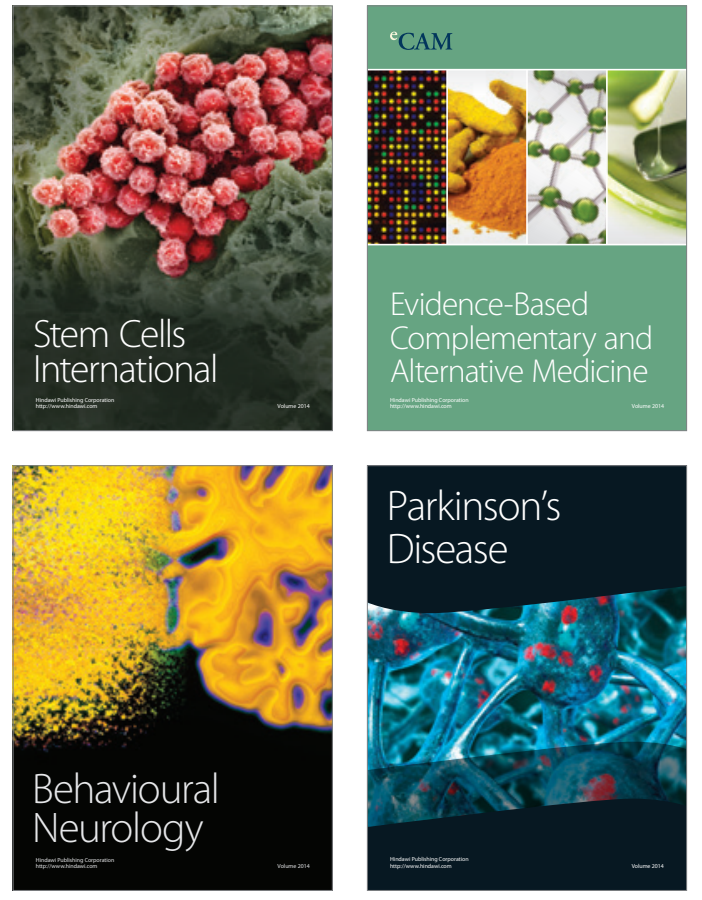
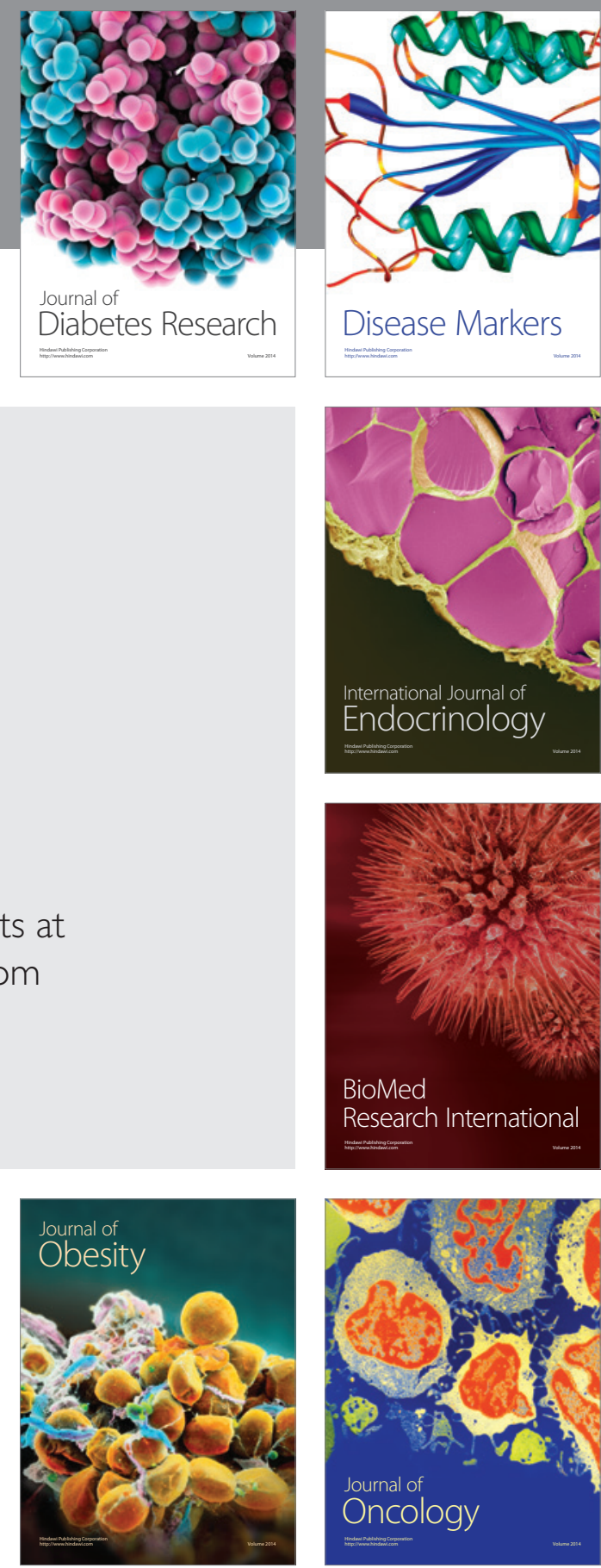

Disease Markers
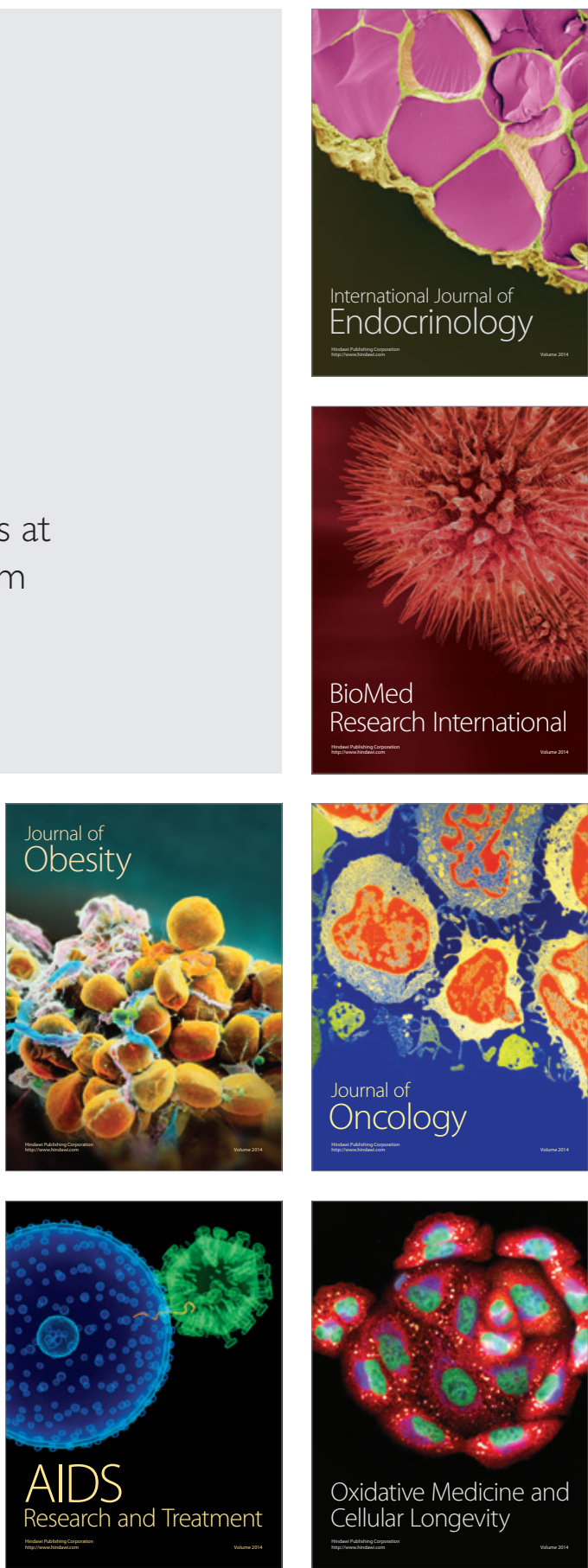